Proc. Estonian Acad. Sci. Eng., 2003, 9, 1, 3-24

\title{
Exploring irregular vibrations and chaos by the wavelet method
}

\author{
Ülo Lepik \\ Institute of Applied Mathematics, University of Tartu, Liivi 2, 50409 Tartu, Estonia; ylepik@ut.ee \\ Received 8 October 2002

\begin{abstract}
The paper cosiders possibilities of exploring nonlinear structural vibrations and detecting chaos by the wavelet method. The signal is decomposed into several lower resolution components. Some numerical quantities for characterizing the signal (wavelet energy distribution, Shannon and threshold entropy, similarity index, self-similarity) are introduced. Noisy systems are discussed. An example, in which the noise transfers chaotic motion to regular vibrations, is described. The wavelet packet method is applied; it is shown that such an approach allows to decrease the width of the frequency bands and gives the possibility to distinguish chaotic vibrations from random motion.
\end{abstract}

Key words: non-linear vibrations, chaos, wavelet method, entropy, noisy systems.

\section{INTRODUCTION}

The development of the wavelet method has been extraordinary fast. This technique, which was introduced by Morlet and Meyer in early 1980s, has turned now to a well-grounded and powerful mathematical tool. This topic has been treated in many textbooks from which we would recommend here $\left[{ }^{1-3}\right]$. This paper mainly considers the wavelet analysis of vibrations in structural dynamics. In this field many interesting papers have been published; to be short we shall refer here only to some recent papers $\left[{ }^{4-6}\right]$.

The wavelet method could be also a valuable tool for analysing chaotic motions, but surprisingly there are very few papers written on this topic. Permann and Hamilton $\left[{ }^{7}\right]$ examined chaotic vibrations of the Duffing attractor; making use of the Daubechies wavelet transform, the coefficients for different resolution levels were computed. Zheng et al. $\left[{ }^{8}\right]$ analysed vibrations of a cracked rotor system with the aid of the Newland wavelets; to demonstrate chaotic effects, the amplitudes of the frequency bands versus time were plotted. Wong and Chen $\left[{ }^{9}\right]$ considered the case in which the frequency of harmonic 
vibrations changes during the motion. Using the Morlet wavelets, modulus and phase diagrams were plotted. Ioussoupov et al. $\left[{ }^{10}\right]$ integrated the Maxwell-Ploch equations for two-level atoms. The motion was analysed with the aid of the Morlet wavelets and structural Hamiltonian chaos was demonstrated. Jubran et al. $\left[{ }^{11}\right]$ examined dynamic response of the flow induced vibrations applying again the Morlet wavelets. The authors of $\left[{ }^{11}\right]$ drew the conclusion that the wavelet approach reveals more clearly than the classical methods the possible route to chaos. Huang and $\mathrm{Xu}\left[{ }^{12}\right]$ proposed a method for extracting chaotic signals with the aid of different wavelet scales. In the paper by Bukkapatnam et al. $\left[{ }^{13}\right]$, a modified wavelet method for separation of chaotic signals was developed.

As a touchstone of new approaches concerning chaotic motion, the wellstudied Duffing, Lorenz, and Van-der-Pol attractors are often employed. There are numerous papers on this topic and this trend continues $\left[{ }^{14-16}\right]$. Noteworthy are also papers $\left[{ }^{17-18}\right]$. In $\left[{ }^{17}\right]$ it is shown that slow increase in time of the parameters of the Duffing equation may bring to chaotic motion. In $\left[{ }^{18}\right]$ the same effect is attained by varying the external force as a control parameter.

In several papers chaotic behaviour of noisy structures (i.e., structures with random parameters) has been discussed. In many cases the computations were carried out for the Duffing equation or for its modifications $\left[{ }^{19-26}\right]$. We shall analyse these papers in Section 5.

In the case of noisy systems, the question, which is the relationship between chaotic and random motion, arises. This problem was dealt with by SzemplińskaStupnicka $\left[{ }^{27}\right]$, who wrote: "Can chaotic motion be interpreted as nonstationary free motion with randomly modulated amplitude and phase?". We shall try to answer this question in Section 5.

The main goal of this paper is to give an overview of the wavelet methods which can be used for analysing irregular, chaotic, and noisy motions; several numerical examples are presented. The paper is organized in the following way. In Section 2 the theoretical background of the wavelet method is briefly described. The decomposition technique is presented in Section 3. In Section 4 some numerical characteristics of the motion are proposed. Section 5 is devoted to the analysis of noisy systems. Possibilities of the wavelet packet transforms are demonstrated in Section 6. In Section 7 the wavelet method is compared with the classical Fourier method and advantages and shortcomings of both approaches are discussed. For computing the examples and for plotting the diagrams, the Matlab and Mathcad wavelet toolboxes $\left[{ }^{28-29}\right]$ have been used.

\section{THEORETICAL BACKGROUND}

The function $g=g(t)$ is called a mother wavelet if the admissibility condition

$$
\int_{-\infty}^{+\infty} \frac{\hat{g}(\omega)^{2}}{\omega} \mathrm{d} \omega<\infty
$$


where $\hat{g}(\omega)$ is the Fourier transform of $g(t)$, is fulfilled. The wavelet family for $g(t)$ is defined as

$$
g^{*}(t, a, b)=\frac{1}{\sqrt{a}} g\left(\frac{t-b}{a}\right)
$$

Here $a$ is the scale (dilation) parameter and $b$ is the translation parameter. We shall consider only orthogonal wavelets, for which

$$
\int_{-\infty}^{+\infty} g^{*}\left(t, a_{1}, b_{1}\right) g^{*}\left(t, a_{2}, b_{2}\right) \mathrm{d} t= \begin{cases}0 & \text { if } a_{1} \neq a_{2} \text { or } b_{1} \neq b_{2}, \\ 1 & \text { if } a_{1}=a_{2} \text { and } b_{1}=b_{2} .\end{cases}
$$

Let $s=s(t)$ be a time series (signal), for which

$$
E=\int_{-\infty}^{+\infty}|s|^{2} \mathrm{~d} t<\infty
$$

Here $E$ stands for the signal energy.

The wavelet transform of $s(t)$ is

$$
W(a, b)=\frac{1}{\sqrt{a}} \int_{-\infty}^{+\infty} s(t) g\left(\frac{t-b}{a}\right) \mathrm{d} t .
$$

For the most of the wavelet families, a scaling function $\varphi(t)$ is introduced; it is calculated with an iterative algorithm. If the scaling function is known, then using it the wavelet function $\psi(t)$ can be put together (for details see, e.g., $\left[{ }^{1}\right]$ ). The formulae (1)-(5) hold if we take $g=\varphi$ or $g=\psi$.

In the following we shall consider only dyadic scales for which $a=2^{j}$ and $b=k a$. Now the functions $\varphi^{*}$ and $\psi^{*}$ obtain the form

$$
\begin{aligned}
& \varphi^{*}(t, J, k)=\sqrt{2^{-J}} \varphi\left(2^{-J} t-k\right), \\
& \psi^{*}(t, j, k)=\sqrt{2^{-j}} \psi\left(2^{-j} t-k\right),
\end{aligned}
$$

where $j=1, \ldots, J$ denotes the level (order) of the transform.

There are several wavelet families (e.g., Haar, Daubechies, Symmlet, and Bspline wavelets). In Fig. 1, the scaling function and mother wavelet for the Daubechies and Symmlet wavelets of order 8 are plotted. The type of the wavelet must be chosen so that it is consistent with the type of features present in the time series $s=s(t)$. Since in this paper mostly non-linear vibrations are examined, we have chosen the Symmlet wavelet, which is nearly symmetric.

It follows from the wavelet theory that the signal $s=s(t)$ can be expanded into the series

$$
s(t)=\sum_{k} a_{J, k} \varphi_{J, k}(t)+\sum_{j=1}^{J} \sum_{k} d_{j, k} \psi_{j, k}(t)
$$


(a)

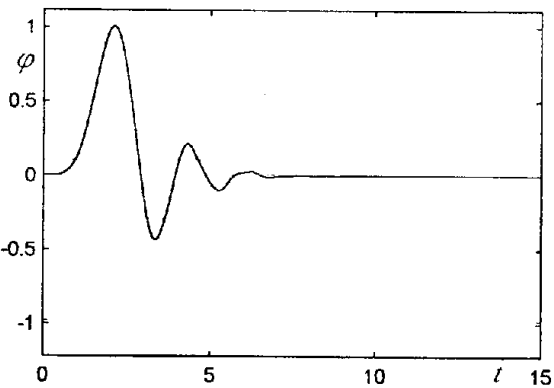

(b)

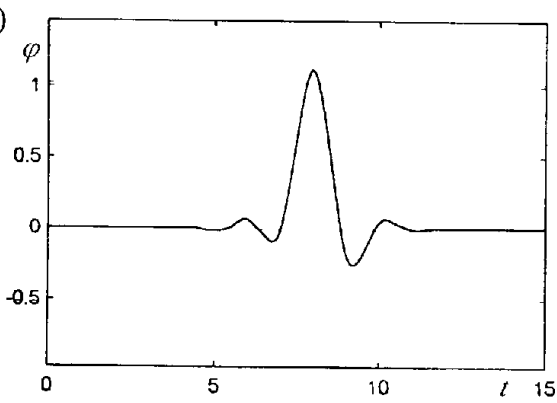

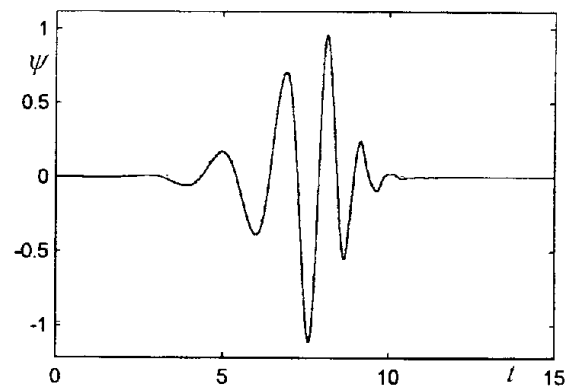

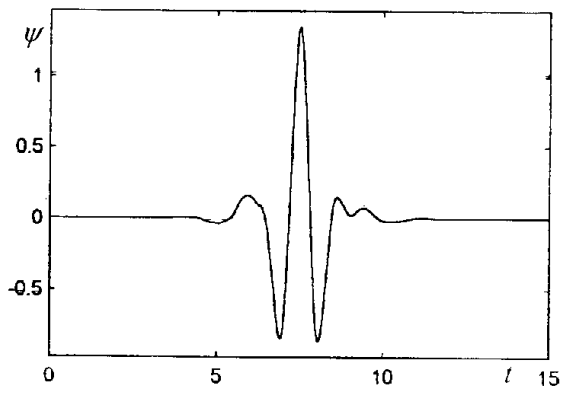

Fig. 1. Wavelets of order 8: (a) Daubechies wavelet; (b) Symmlet wavelet.

where $J$ is the maximal level of resolution, $a_{J, k}$ and $d_{j, k}(j=1, \ldots, J)$ are called wavelet transform coefficients. Due to the orthogonality of the scaling and wavelet functions, these coefficients can be calculated from the formulae

$$
a_{J, k}=\int_{-\infty}^{+\infty} s(t) \varphi_{J, k}(t) \mathrm{d} t, \quad d_{j, k}=\int_{-\infty}^{+\infty} s(t) \psi_{j, k}(t) \mathrm{d} t .
$$

Introducing notations

$$
a_{J}(t)=\sum_{k} a_{J, k} \varphi_{J, k}(t), \quad d_{j}(t)=\sum_{k} d_{j, k} \psi_{j, k}(t),
$$

we can put (7) into the form

$$
s(t)=a_{J}(t)+\sum_{j=1}^{J} d_{j}(t)
$$

This is the decomposition of the signal into orthogonal components. The function $a_{J}(t)$ is the low-frequency component which is called approximation and functions $d_{j}(t)$ are called details of the level $j$. In the signal processing theory the functions $a_{J}$ and $d_{j}$ correspond to the low-pass and high-pass filter, respectively.

Now let us calculate the signal energy $E$. Taking into account the orthogonality of the scaling and wavelet functions, we find from (9)-(10): 


$$
E=E_{a}+\sum_{j=1}^{J} E_{j}
$$

where $E_{a}=\sum_{k} a_{J, k}^{2}, E_{j}=\sum_{k} d_{j, k}^{2}$.

Here $E_{a}$ is the approximation energy and $E_{j}$ is energy of the detail signal at the resolution level $j$.

\section{DECOMPOSITION}

For analysing the time-frequency properties of the signal, it is expedient to decompose the signal into many resolution components. In this section the decomposition is accomplished with the aid of the Matlab Wavelet Toolbox interface $\left[{ }^{28}\right]$. Quite important is the choice of the decomposition level $J$. If $J$ is too big, we get unreasonable results; on the other hand, if $J$ is too small, the resolution of the signal will be insufficient. Maximal value of $J$ depends on the length of the signal and of the type of the chosen wavelet; it can be calculated using the command WMAXLEV in $\left[{ }^{28}\right]$. If the signal length is 512 and we use the Symmlet wavelet of degree 8, we find $J_{\max }=5$.

In the case of the following examples, the signal is obtained by integrating differential equations. This is carried out with the fourth-order Runge-Kutta method with an accuracy of $10^{-6}$. In this Section, from the solution achieved by integration, we have taken out $2^{9}=512$ points, which are uniformly distributed along the $t$ axis.

As the first example let us consider the Duffing equation

$$
\frac{\mathrm{d}^{2} x}{\mathrm{~d} t^{2}}+r \frac{\mathrm{d} x}{\mathrm{~d} t}-x+x^{3}=g \cos \omega t, \quad 0 \leq t \leq 200,
$$

with the initial conditions

$$
x(0)=0, \quad \frac{\mathrm{d} x}{\mathrm{~d} t}(0)=1 .
$$

In Fig. 2, the decomposition plot is presented for $r=0.25, \omega=1, g=0.3$. In this figure $a_{5}$ denotes the approximation at $J=5, d_{5}-d_{1}$ are details for different decomposition levels. The original signal $s$ can be synthesized if we add all these terms: $s=a_{5}+d_{5}+d_{4}+d_{3}+d_{2}+d_{1}$. It follows from Fig. 2 that the signal consists of several components (only the influence of $d_{1}$ can be neglected).

Multilevel decomposition is especially valuable in the cases in which the nature of the external loading changes during the motion. As an example we shall consider the case in which $r=0.15, \omega=1$, and

$$
g=\left\{\begin{array}{cl}
3 & \text { for } 80 \leq t \leq 120 \\
0.3 & \text { otherwise }
\end{array}\right.
$$



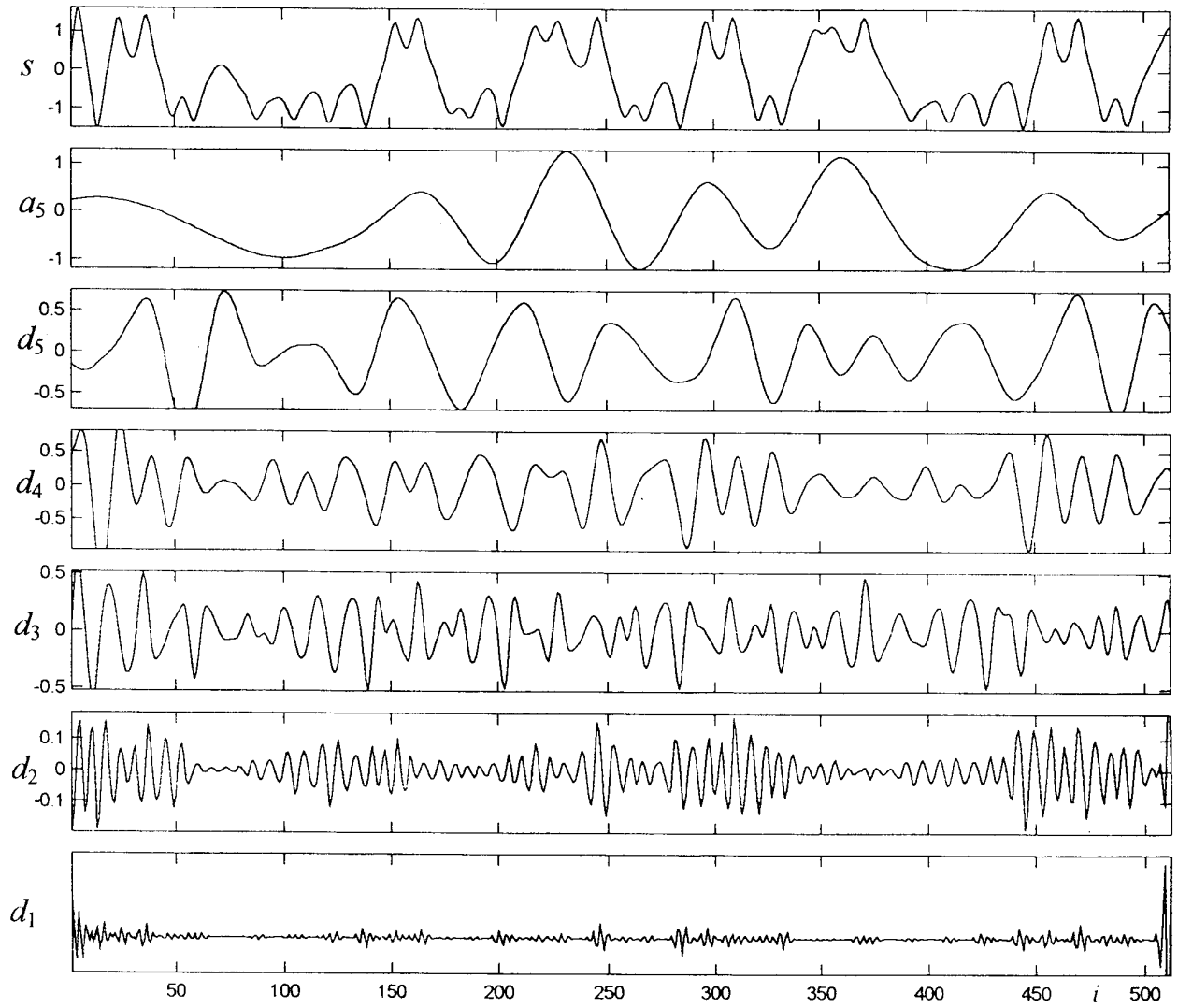

Fig. 2. Decomposition for the Duffing equation with $r=0.25, \omega=1, i=512 t / T$.

Decomposition plots for this case are shown in Fig. 3. For $0 \leq t \leq 80$ the motion is practically regular and the detail $d_{3}$ dominates. In the interval $80<t<120$ higher frequencies appear and the motion is assumed to be chaotic. For $120<t<200$ only the components $a_{5}, d_{5}$, and $d_{4}$ are essential and the motion is quasiperiodic.

In the next example we shall examine the chaotic Lorenz attractor:

$$
\begin{aligned}
& \frac{\mathrm{d} x}{\mathrm{~d} t}=-10(x-y), \\
& \frac{\mathrm{d} y}{\mathrm{~d} t}=r x-y-x z, \quad(0 \leq t \leq 100), \\
& \frac{\mathrm{d} z}{\mathrm{~d} t}=x y-\frac{8}{3} z,
\end{aligned}
$$



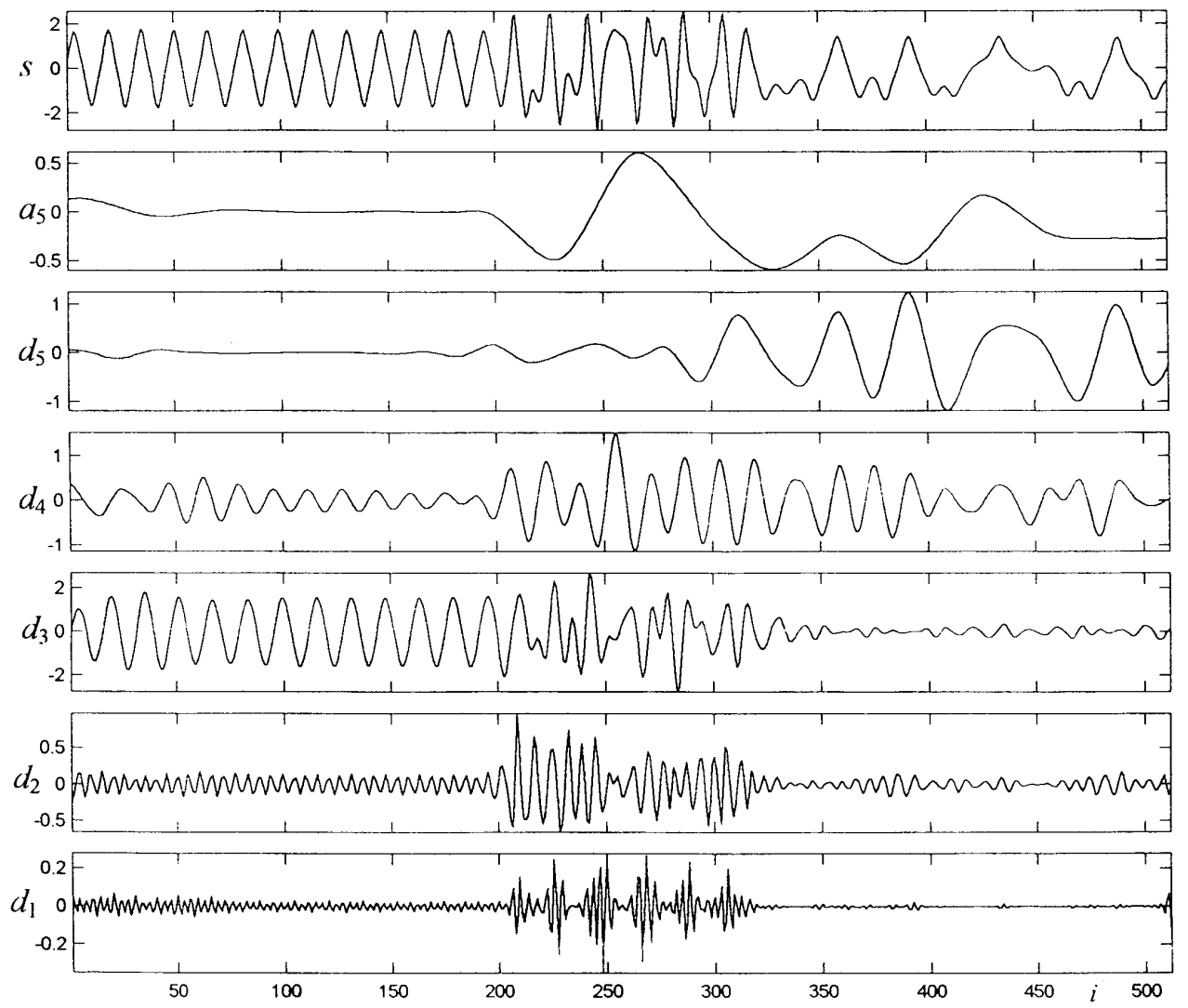

Fig. 3. Decomposition for Duffing equation with $r=0.15, \omega=1$; amplitude of the external loads is calculated according to (13).

with initial conditions $x(0)=0, y(0)=0.1$, and $z(0)=0.1$. Decomposition for the $x$ component is carried out for $r=28$ and the results are plotted in Fig. 4 . Here the details $d_{3}-d_{1}$, which correspond to higher frequencies, are more important than in Figs. 2-3.

There are also other possibilities for analysing the signal in the timefrequency plane. One of them is to put together the mean square map $\left[{ }^{1}\right]$, pp. 349-353. In the case of dyadic wavelets of order $j$ we have $l(j)=2^{j}$ wavelet coefficients ${ }^{1}$ in the interval $t \in[0, T]$. Let us divide this interval into $l(j)$ boxes of uniform length; in the centers of the boxes we space the values $\left[d_{j}(k)\right]^{2}$, where $k=1,2, \ldots, l(j)$.

A contour plot of these squares maps the distribution of the wavelet energy between different levels and different positions. Such a diagram for the

${ }^{1}$ In some textbooks and also in the manual $\left[{ }^{28}\right]$ downsampling has been used (passing to lower levels of decomposition every second data point is thrown away). In this case the number of wavelet coefficients $l(j)$ somewhat deviates from $2^{j}$. 
$x$ component of the Lorenz attractor with $r=24.5$ is plotted in Fig. 5 (for that purpose the program MAPDN from [ $\left.{ }^{1}\right]$ has been applied). Of course the contour diagram can be replaced at will with a 3-dimensional plot.
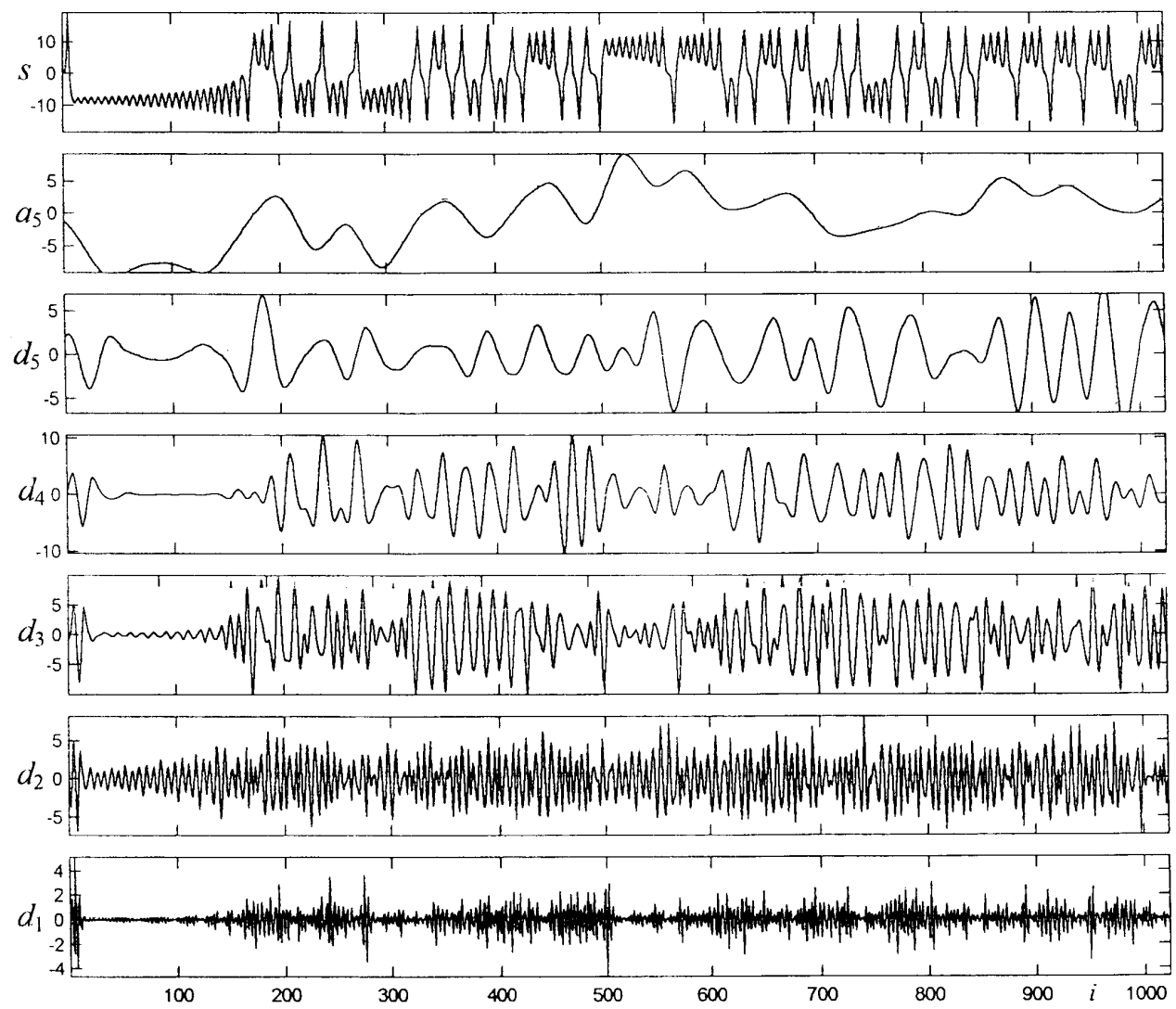

Fig. 4. Decomposition for Lorenz equation with $r=28, \quad i=512 t / T$.

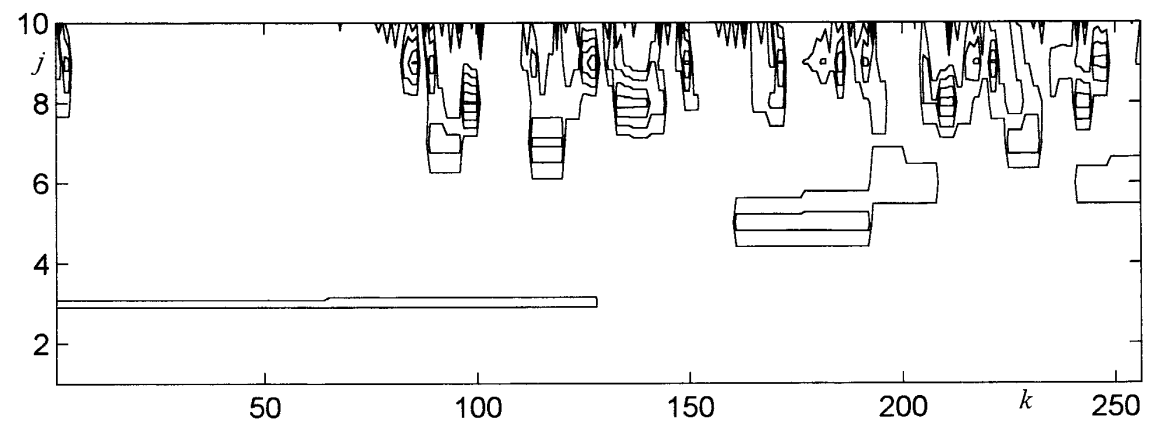

Fig. 5. Mean square map for the Lorenz equation with $r=24.5$. 
The decomposition method gives an overview how the frequencies are distributed by levels and time. Its shortcoming is that in most cases the localization in frequency is not satisfactory. Therefore it is sometimes difficult to find out visually whether the motion is quasiperiodic or chaotic.

Some possibilities to overcome this difficulty we shall discuss in Sections 4 and 6 .

\section{SOME NUMERICAL CHARACTERISTICS}

In this section we shall consider some numerical characteristics which allow us to describe the main features of the signal $s=s(t)$. One possibility is to find out how the signal energy is distributed between the resolution levels. For this purpose the equations (11) may be used. It is expedient to introduce the relative wavelet energy

$$
p_{J+1}=\frac{E_{a}}{E}, \quad p_{j}=\frac{E_{j}}{E} \quad(j=1,2, \ldots, J) .
$$

Another way is to calculate the Shannon entropy. Following $\left[{ }^{28}\right]$, we define it as

$$
S=-\sum_{j=1}^{J+1} p_{j} \ln p_{j}
$$

The quantity $S$ measures the disorder of the system. As an illustration we shall apply (16) in a simple case in which only the first $k$ terms of the sequence $\left\{p_{j}\right\}$ are different from zero. Let us find such values $p_{j}$ for which the entropy is maximal. Here we have to solve the mathematical problem

$$
S=-\sum_{j=1}^{k} p_{j} \ln p_{j}=\max , \quad \sum_{j=1}^{k} p_{j}=1 .
$$

Making use of the differential calculus we find $p_{1}=p_{2}=\ldots=p_{k}=1 / k$ and $S_{\max }=\ln k$. We see that the system has maximal disorder if all the quantities $p_{j}$ are distributed uniformly. In the case of regular vibrations with the same frequency, we have $k=1$ and $S_{\max }=0$. If $k=2$, then $S_{\max }=0.693$ and for $k=3$ we have $S_{\max }=1.099$.

It can be seen from the decomposition diagrams, presented in Section 3, that in some cases the values of the functions are quite small and may be neglected. In this case the concept of threshold entropy, which is proposed in $\left[{ }^{28}\right]$ (pages 6106) could be useful. Here a threshold value $\varepsilon$ is prescribed and a number of time instants, for which the function values are greater that $\varepsilon$, are evaluated.

If the character of motion changes during the time $t \in[0, T]$, then it is useful to analyse the time evolution of the wavelet energy and entropy. For this purpose we recommend the following procedure which is a modification of the method proposed in $\left[{ }^{30}\right]$. 
Step 1: Calculate the wavelet coefficients for $t \in[0, T]$.

Step 2: Compose the time-frequency map as shown in Section 3.

Step 3: Divide the signal into non-overlapping temporal windows. To be concrete, in the following we shall consider the time window with the endpoints $t_{a}$ and $t_{b}$.

Step 4: Find out which of the wavelet coefficients belong to the interval $\left(t_{a}, t_{b}\right)$. Summing up the squares of these components for the $j$ th decomposition we get $E_{j}$.

Step 5: Calculate $p_{j}$ and $S$ for the given time window.

Next we shall consider the case in which we have two different distributions $\left\{p_{j}\right\}$ and $\left\{q_{j}\right\}$ with $\sum_{j} p_{j}=\sum_{j} q_{j}=1$ (they can be considered as representing the distributions of the wavelet energy for two segments of a signal or two different signals). In $\left[{ }^{28}\right]$ it is recommended to introduce the coefficient

$$
S(p \mid q)=\sum_{j} p_{j} \ln \frac{p_{j}}{q_{j}}
$$

which gives a measure of the degree of similarity between the two distributions. This quantity is always positive and vanishes only if $p_{j} \equiv q_{j}$ for $j=1, \ldots, J$.

An interesting property of non-linear systems is selfsimilarity. A time series is called selfsimilar if the equation

$$
s(t)=a^{-H} s(a t)
$$

holds statistically. Here $a>0$ and $H$ is called the Hurst exponent.

It can be shown $\left[{ }^{31}\right]$ that for selfsimilar signals

$$
E_{j} \approx 2^{(2 H+1) j} \cdot \text { const. }
$$

This result can be written in the form

$$
v_{j}=2^{-j} E_{j}=2^{2 H j} \cdot \text { const. }
$$

It follows from (19) that

$$
\log v_{j}=2 H j \log 2+\text { const. }
$$

Next we shall make the diagram $\left(j, \log v_{j}\right)$ for $j=1,2, \ldots, J$. If the points of this plot lie near a straight line, then the motion can be considered selfsimilar. The slope of this line is $k=2 H \log 2$; thus the Hurst exponent $H$ can be calculated.

This method has been applied by Scargle et al. $\left[{ }^{32}\right]$. As a shortcoming of this method Scargle notes: “... it is not particularly good at detecting periodic signals". A similar method for calculating the Hurst exponent, which is called the rescaled range method, is presented in $\left[{ }^{33}\right]$; it is applicable for arbitrary time series. 
There are several papers $\left[{ }^{34-35}\right]$, in which statistical concepts (such as variance, bias kurtosis, etc.) have been used for analysing transient signals. In this paper the statistical approach is not considered.

Let us consider some examples. We shall start with the Duffing equation (12) for $\omega=1, g=0.3, J=6 ; r$ is the control parameter. The Shannon entropy and relative wavelet energies are plotted in Fig. 6 . It follows from this diagram that the Shannon entropy has a jump at $r=0.174$; here period doubling takes place and the subsequent motion is irregular. For $r<0.174$ the decomposition $j=5$ dominates, after that the role of lower frequencies increases. To illustrate this transition phenomenon, the time histories for three values of the control parameter $r$ are plotted in Fig. 7.

Next, let us pass to the Lorenz system (14). Computer simulation results for the decomposition levels $a_{6}$ and $d_{6}-d_{1}$ are presented in Table 1 . Symbol $T$ denotes threshold entropy and is calculated in the following way. At first, we find the maximum of the signal $x_{\max }$ and for each decomposition find the percentage of the values which are greater than $0.05 x_{\max }$. All calculations were carried out for the $x$ component (except the last row of the Table 1, where results for the $z$ component are shown). Time histories for some interesting cases are plotted in Fig. 8 .
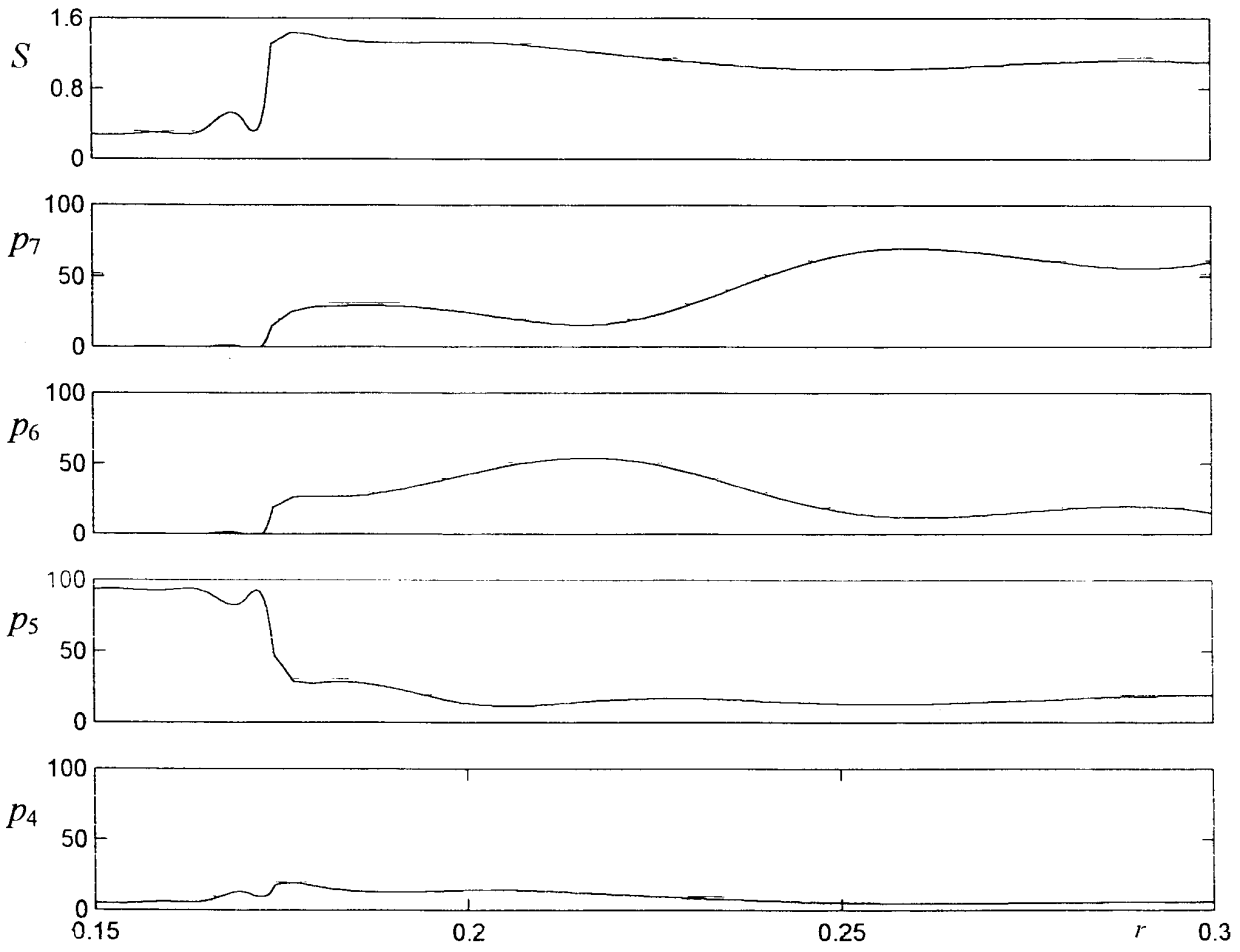

Fig. 6. Duffing equation (12) for $\omega=1, g=0.3, \quad J=6$. Dependence of the Shannon entropy $S$ and relative wavelet energies $p_{7}-p_{4}$ (in percents) on the parameter $r$. 
(a)

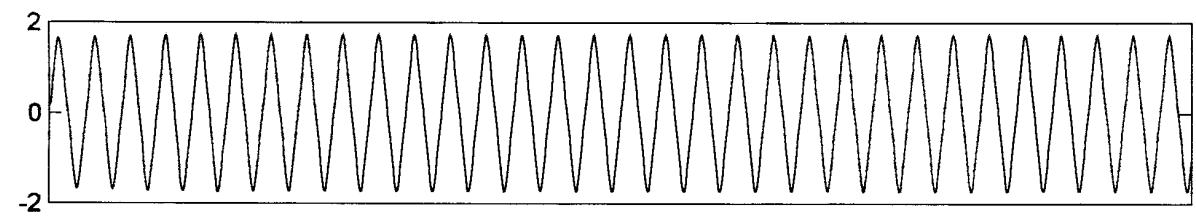

(b)

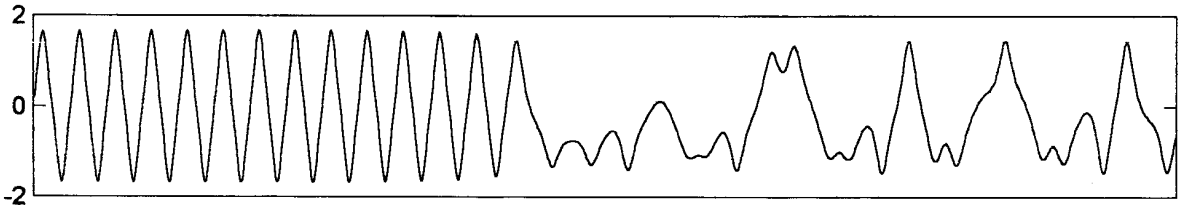

(c)

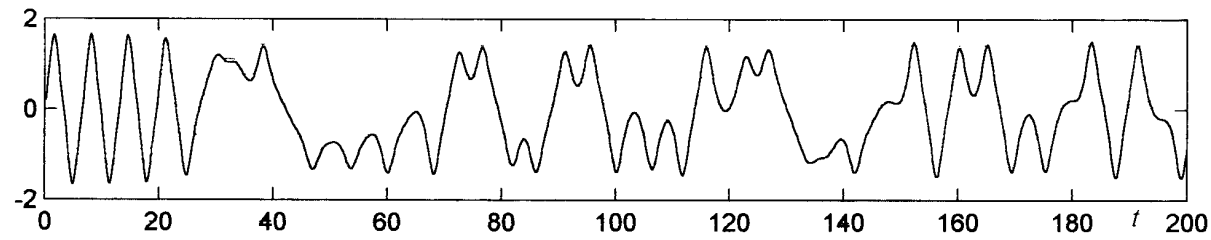

Fig. 7. Time histories of the Duffing attractor for $\omega=1, g=0.3$ : (a) $r=0.17$, (b) $r=0.174$, (c) $r=0.18$

Table 1. Decomposition of the Lorenz system for different values of the control parameter $r$

\begin{tabular}{|c|c|c|c|c|c|c|c|c|c|}
\hline$r$ & $P / T$ & $a_{6}$ & $d_{6}$ & $d_{5}$ & $d_{4}$ & $d_{3}$ & $d_{2}$ & $d_{1}$ & $S$ \\
\hline \multirow[t]{2}{*}{22} & $p$ & 96.7 & 0.9 & 0.7 & 0.6 & 0.6 & 0.6 & 0 & \multirow[t]{2}{*}{0.200} \\
\hline & $T$ & 100.0 & 13.5 & 7.4 & 2.8 & 5.6 & 8.8 & 0.2 & \\
\hline \multirow[t]{2}{*}{24} & $p$ & 94.4 & 0.9 & 0.6 & 0.5 & 1.0 & 2.4 & 0.1 & \multirow[t]{2}{*}{0.297} \\
\hline & $T$ & 100.0 & 13.4 & 7.3 & 2.8 & 12.8 & 55.7 & 0.59 & \\
\hline \multirow[t]{2}{*}{24.5} & $p$ & 37.6 & 10.2 & 8.0 & 15.6 & 18.0 & 9.8 & 0.9 & \multirow[t]{2}{*}{1.670} \\
\hline & $T$ & 86.8 & 65.3 & 51.6 & 56.0 & 69.1 & 75.9 & 19.1 & \\
\hline \multirow[t]{2}{*}{28} & $p$ & 25.5 & 5.3 & 12.6 & 20.4 & 23.4 & 11.4 & 1.4 & \multirow[t]{2}{*}{1.7358} \\
\hline & $T$ & 78.5 & 60.9 & 70.4 & 71.3 & 74.0 & 76.0 & 25.0 & \\
\hline \multirow[t]{2}{*}{32} & $p$ & 17.8 & 7.5 & 17.6 & 18.8 & 22.7 & 13.7 & 1.93 & \multirow[t]{2}{*}{1.806} \\
\hline & $T$ & 79.8 & 63.3 & 75.1 & 70.8 & 72.8 & 74.5 & 31.1 & \\
\hline 166.3 & $p$ & 0.2 & 0.3 & 2.9 & 27.2 & 39.0 & 28.0 & 2.5 & \multirow[t]{2}{*}{1.3012} \\
\hline$x$ comp & $T$ & 3.8 & 1.9 & 34.9 & 79.4 & 81.4 & 80.8 & 33.9 & \\
\hline 166.3 & $p$ & 93.5 & 0.9 & 0.5 & 0.3 & 2.2 & 1.8 & 0.2 & 0.346 \\
\hline$z$ comp & $T$ & 100.0 & 13.4 & 5.1 & 5.9 & 69.2 & 64.5 & 32.6 & \\
\hline
\end{tabular}

It follows from Table 1 and Fig. 8 that Shannon entropy has a jump between $r=24$ and $r=24.5$ (here a limit cycle becomes instable). At $r=166.3$ solutions for the $x$ and $z$ component are completely different. Interesting is the behaviour of the $z$ component. Low value of the Shannon entropy $(S=0.346)$ 
(a)

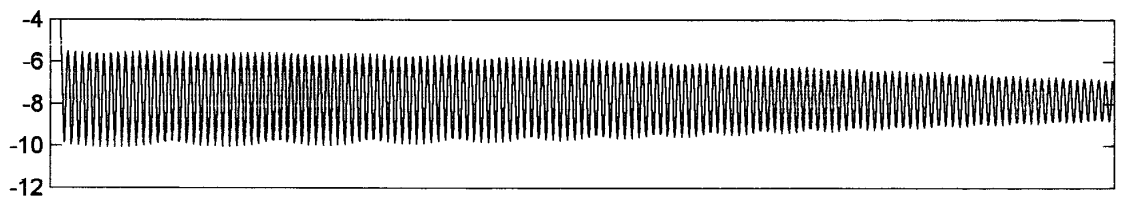

(b)

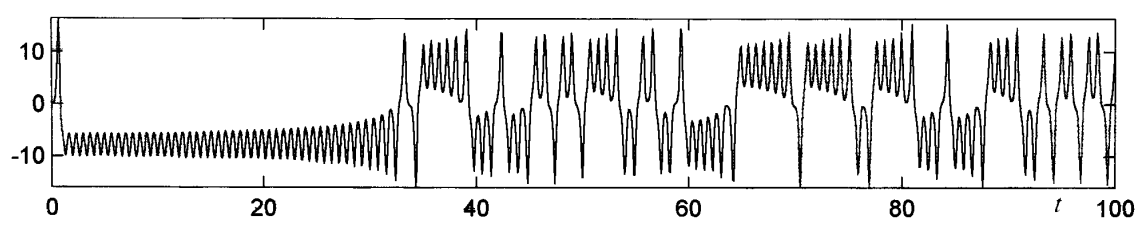

(c)

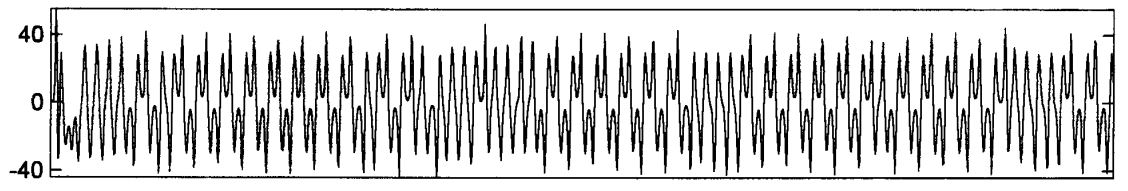

(d)

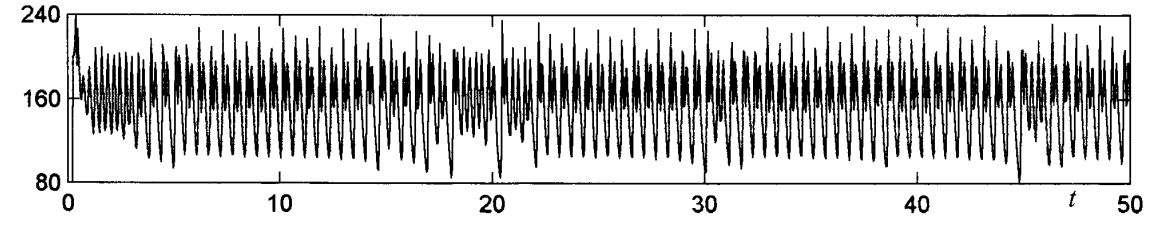

Fig. 8. Time histories of the Lorenz attractor: (a) $r=24, \quad$ (b) $r=24.5, \quad$ (c) $r=166.3$ ( $x$ component), (d) $r=166.3$ ( $z$ component).

and the fact that most of the wavelet energy belongs to the approximation $a_{6}$ indicates regularity in motion. On the other hand, the threshold entropy analysis shows high values for the decompositions $d_{3}-d_{1}$ (which carry only an insignificant part of the wave energy). All this leads to chaotic outbreaks at irregular intervals. This phenomenon was described by Manneville and Pomeau $\left[{ }^{36}\right]$ and is called intermittency.

Szemplińska-Stupnicka $\left[{ }^{27}\right]$ analysed the Duffing-Van-der-Pol equation

$$
\frac{\mathrm{d}^{2} x}{\mathrm{~d} t^{2}}-0.2\left(1-x^{2}\right) \frac{\mathrm{d} x}{\mathrm{~d} t}+x^{3}=12 \cos v t
$$

with the control parameter $v$. Her theoretical analysis showed that in the interval $3.5<v<4.2$ the subharmonic frequencies $v / 2$ and $v / 3$ appear; in the transition zone the motion is chaotic. Let us discuss this problem using the wavelet method. Computer simulation results are shown in Table 2. The Shannon entropy has three maxima: $S=1.182$ at $v=3.82, S=1.126$ at $v=4.09$, and $S=1.122$ at $v=4.19$, which correspond to the transition frequencies. If $v$ increases, the maximal values of the wavelet energy move to lower decompositions. These conclusions completely confirm the results of $\left[{ }^{27}\right]$. 
Table 2. Decomposition of the Duffing-Van-der-Pol system for different values of the parameter $v$

\begin{tabular}{c|c|c|c|c|c|c|l}
\hline$v$ & $p_{7}$ & $p_{6}$ & $p_{5}$ & $p_{4}$ & $p_{3}$ & $p_{2}$ & \multicolumn{1}{c}{$S$} \\
\hline 3.5 & 1.9 & 1.0 & 11.7 & 73.7 & 11.6 & 0.1 & 0.8534 \\
3.6 & 2.2 & 2.0 & 19.6 & 63.6 & 12.5 & 0.1 & 1.036 \\
3.7 & 1.7 & 2.7 & 21.8 & 60.5 & 13.2 & 0.2 & 1.083 \\
3.8 & 2.7 & 1.9 & 28.6 & 53.3 & 13.3 & 0.1 & 1.141 \\
3.9 & 2.1 & 2.0 & 29.3 & 50.8 & 15.6 & 0.1 & 1.164 \\
4.0 & 1.8 & 1.0 & 54.3 & 26.1 & 16.8 & 0.0 & 1.105 \\
4.1 & 1.4 & 1.4 & 51.9 & 29.6 & 15.7 & 0.1 & 1.119 \\
4.2 & 0.1 & 0.8 & 70.1 & 14.9 & 14.5 & 0.0 & 0.8570
\end{tabular}

To illustrate time evolution of the method let us return to Fig. 4, where decomposition of the Duffing equation is plotted. Since the coefficient $g$ is prescribed according to (13), the motion consists of three different parts. Making use of the algorithm presented above, we have calculated the wavelet energies and Shannon entropy both for the whole signal and for its segments. The results are presented in Table 3. It follows from this table that most irregular is the motion in the interval $t \in(120,200)$.

Next, let us give an example about the degree of similarity between two distributions. We shall consider the Lorenz equation (14) for $r=24.5$ and $T=100$. We shall compare here the segments $t \in[0,50]$ and $t \in[50,100]$. The Shannon entropy for these segments is $S_{1}=1.309$ and $S_{2}=1.796$. Applying the formula (17), we find $S(p \mid q)=0.697$. Carrying out the same computations for $r=28$, we get $S_{1}=1.633, S_{2}=1.698$, and $S(p \mid q)=0.243$. Consequently, the segments in the case $r=28$ are more similar than for $r=24.5$. If we compare the segments $t \in[50,100]$ for $r=24.5$ and for $r=28$, we find $S(p \mid q)=0.111$.

The last example is about the selfsimilarity of the Duffing attractor for $r=0.25$ and $J=8$. Making use of (20) we make the $\left(j, \log v_{j}\right)$ diagram, which is shown in Fig. 9a. Since all the calculated points are nearly on a straight line, the motion may be regarded as selfsimilar. The slope of this straight line is 0.136 and the Hurst exponent is 0.226 .

Table 3. Wavelet energy and Shannon entropy for the Duffing attractor shown in Fig. 4

\begin{tabular}{c|r|c|c|c|c|c|c}
\hline Time interval & $p_{7}$ & $p_{6}$ & $p_{5}$ & $p_{4}$ & $p_{3}$ & $p_{2}$ & $S$ \\
\hline $0 \leq t<80$ & 0.3 & 0.3 & 88.9 & 9.7 & 0.8 & 0.1 & 0.419 \\
$80 \leq t<120$ & 6.4 & 1.5 & 35.6 & 50.9 & 5.3 & 0.3 & 1.126 \\
$120 \leq t \leq 200$ & 21.0 & 9.3 & 48.7 & 19.8 & 0.9 & 0.1 & 1.270 \\
$0 \leq t \leq 200$ & 8.8 & 4.8 & 51.2 & 32.0 & 3.1 & 0.2 & 1.186
\end{tabular}



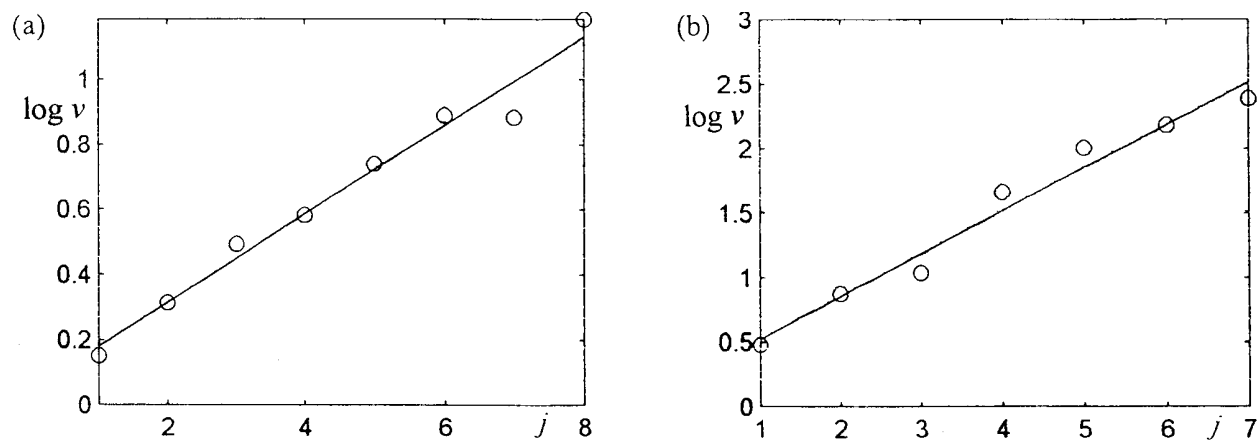

Fig. 9. Selfsimilarity of the Duffing attractor for $r=0.25$ (a) and fractional Brownian motion (b).

\section{NOISY SYSTEMS}

The first question which arises is how to distinguish chaotic motion from a stochastic signal. In the case of zero-mean Gaussian white noise, spectral density of the wavelet power, defined by (15), is independent of time and scale $\left[{ }^{37}\right]$. Thus if at all levels we encounter signals, which are clearly irregular, and higher frequencies carry most of the energy, the motion may be regarded as random.

An important class among the random motions is the fractional Brownian motion. It is a selfsimilar motion with the Hurst exponent $0<H<1$. It can be shown $\left[{ }^{38}\right]$ that the equation $D=2-H$, where $D$ is the fractal dimension, holds. If $H=0.5$, we get the ordinary Brownian motion.

The Hurst exponent can be calculated with the methods indicated in Section 4. As an example, let us analyse the fractional Brownian motion, which was calculated according to the method described in $\left[{ }^{29}\right]$. Decomposition of this motion gave the following results: $p_{8}=21.1, p_{7}=13.3, p_{6}=17.5, p_{5}=15.8$, $p_{4}=7.4, p_{3}=10.4, p_{2}=8.4, p_{1}=5.9$; the Shannon entropy was $S=0.868$. Making use of (20), we get the diagram shown in Fig. 9b; it shows that $H=0.55$.

Behaviour of noisy systems (some parameters are random) is investigated in several textbooks (e.g., $\left.{ }^{39}\right]$ ) and papers. It is interesting to note that in several papers the stochastic Duffing oscillator has been investigated $\left.{ }^{20-25}\right]$. The randomness has been introduced in different ways. In $\left[{ }^{24}\right]$ the forcing term of the equation (12) was taken in the form $F(t)=g \cos \omega t+\varepsilon \xi(t)$, where $\xi(t)$ is a zero mean unit Gaussian process and $\varepsilon>0$ is a small parameter. In $\left[{ }^{22}\right]$ the forcing term has the form $F(t)=(1-\gamma) g \cos \omega t+\gamma \xi(t)$, where $0 \leq \gamma \leq 1$. In $\left[{ }^{20}\right]$ it is assumed that $F(t)$ is a narrow-band stationary random process. The coefficient of the linear term $x$ is randomized in $\left[{ }^{21,23,25}\right]$. Wavelet method for analysing noisy systems has been applied in $\left[{ }^{26}\right]$.

In these papers, computer simulation for calculating Ljapunov exponents, phase diagrams, and Poisson maps has been applied; also the Melnikov method was used. The main results are as follows. The random noise will change the 
response of the system from a limit cycle to a diffused limit cycle. The Poincaré maps are diffused and smeared, they occupy larger area of the phase plane. Periodic windows of the largest Ljapunov exponent are gradually washed out or diminished. The value of the amplitude of periodic forcing for the onset of the chaotic motion is reduced with the increase of the noise intensity. If the deterministic system is regular, then noise may induce chaos.

We shall investigate the Duffing attractor (12), assuming that the amplitude of the forcing term and angular velocity are functions of the narrow-band Gaussian random variables $\xi(t)$ :

$$
\begin{aligned}
& g(t)=g_{0}[1+\alpha \xi(t)], \\
& \omega(t)=\omega_{0}[1+\beta \xi(t)] .
\end{aligned}
$$

Here $\alpha, \beta \in[0,1]$ are prescribed coefficients, characterizing the noise intensity. We shall consider the case in which the motion for $\alpha=\beta=0$ is chaotic. Computer results for $r=0.25$ are plotted in Fig. 10 (it should be mentioned that the noise essentially increases the computing time; if we want to achieve the same precision as in the case of the deterministic system, then the computer time will be increased for 100-200 times).

(a)

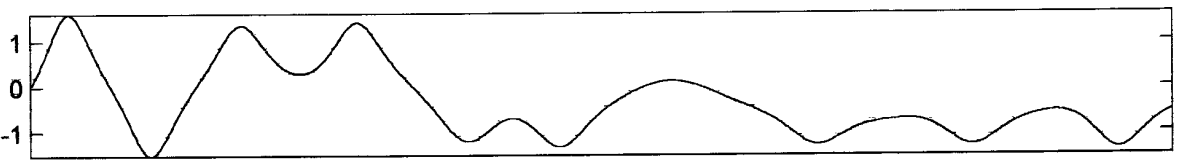

(b)

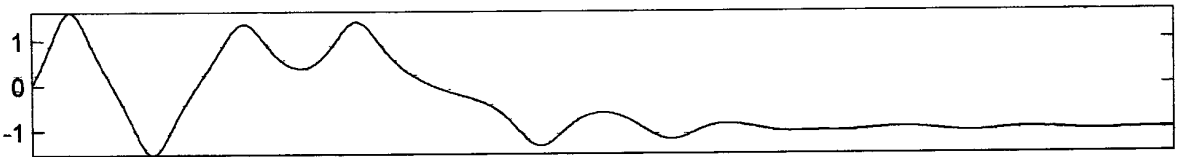

(c)

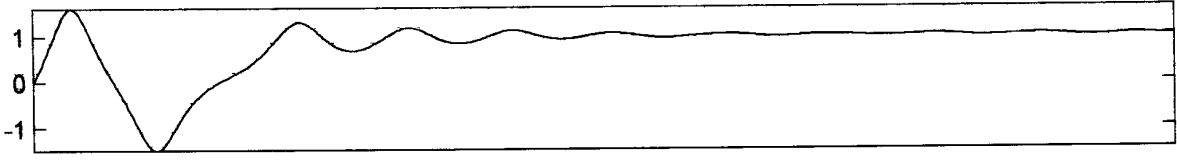

(d)

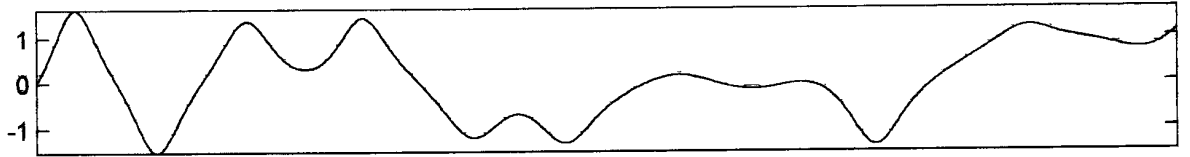

(e)

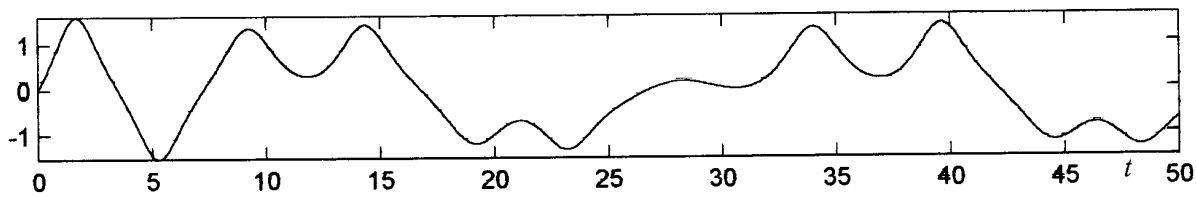

Fig. 10. Time histories of the Duffing equation for $r=0.25$ : (a) deterministic case; stohhastic cases: (b) $\alpha=0, \beta=0.05$; (c) $\alpha=0, \beta=0.2$; (d) $\alpha=0.05, \beta=0$; (e) $\alpha=0.2, \beta=0$. 
Especially interesting are the cases $b$ and $c$ with $\beta \neq 0$ (angular velocity is random). Here the chaotic motion with increasing $t$ becomes regular and is terminated in one of the focuses $x= \pm 1$. This conclusion is confirmed by decomposition analysis. For $\beta=0.2$ we get $p_{7}=92.8, p_{6}=6.6, p_{5}=0.5$, $p_{4}=0, S=0.285$ (in the deterministic case we would have $p_{7}=64.9$, $\left.p_{6}=16.4, p_{5}=13.1, p_{4}=5.8, S=1.191\right)$. This is an interesting result and deserves further investigation.

Summing up what was said above, we see that the effect of the noise to nonlinear vibrations is dual: it can induce chaos or it may smooth the motion and transmute it from the chaotic state to regular vibrations. This is also the answer to the question which was raised by Szemplińska-Stupnicka $\left[{ }^{27}\right]$ and was formulated in Section 1.

In the case of noisy systems the effect of the noise may be eliminated; for this purpose several denoising programs are available $\left[{ }^{28-29}\right]$.

\section{WAVELET PACKETS}

Wavelet packet transform was introduced by Coifman and Wickerhauser $\left[{ }^{40}\right]$ in early 1990s. Wavelet packet functions are superpositions of the wavelet functions, which are defined by the following sequence of functions:

$$
W_{2 n}(t)=2 \sum_{k=0}^{2 N-1} h(k) W_{n}(2 t-k), \quad W_{2 n+1}(t)=2 \sum_{k=0}^{2 N-1} g(k) W_{n}(2 t-k) .
$$

Here $W_{0}(t)=\varphi(t)$ is the scaling function and $W_{1}(t)=\psi(t)$ is the wavelet function, $g(k)$ and $h(k)$ denote the coefficients of the scaling function and the wavelet, respectively. A three-indexed family of analysing functions is put together

$$
W_{j, n, k}(t)=2^{-\frac{j}{2}} W_{n}\left(2^{-j} t-k\right),
$$

where $j$ is the level of decomposition and $k$ is the position. The signal $s(t)$ is expanded in the following way

$$
s(t)=\sum_{j} \sum_{n} \sum_{k} a_{j n k} w_{j, n, k}(t)
$$

where

$$
a_{n j k}=\int_{-\infty}^{+\infty} s(t) W_{j, n, k}(t) \mathrm{d} t
$$

To explain this method, let us consider the binary tree of the wavelet and wavelet packet transform (Fig. 11). In the case of the wavelet method, each approximation is split into a second-level approximation and detail (Fig. 11a). 
(a)

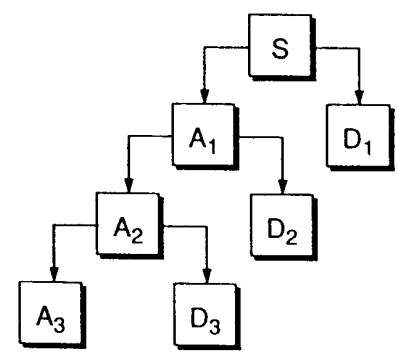

$$
\begin{aligned}
S & =A_{1}+D_{1} \\
& =A_{2}+D_{2}+D_{1} \\
& =A_{3}+D_{3}+D_{2}+D_{1}
\end{aligned}
$$

(b)

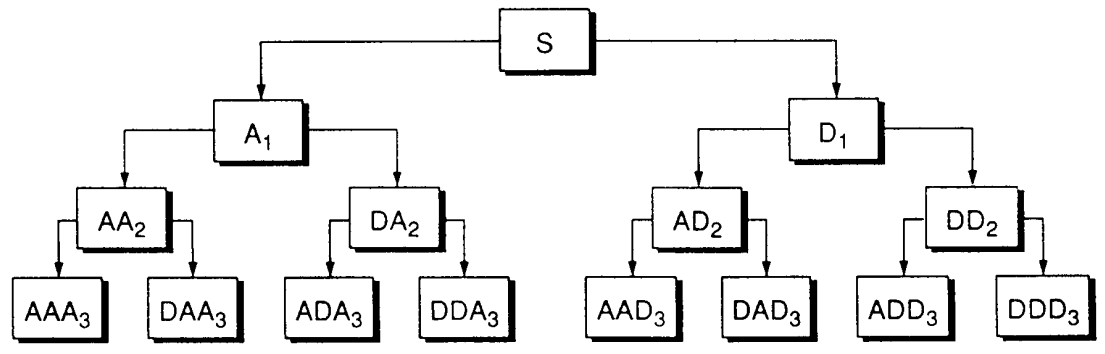

Fig. 11. Binary trees for the wavelet transform (a) and the wavelet packet transform (b); $S$ - signal, $\mathrm{A}_{i}$ and $\mathrm{D}_{i}$ denote approximations and decompositions, respectively.

For a $j$-level decomposition there are $j+1$ possible ways to decompose the signal. In the wavelet packet analysis, the details as well the approximations are split (Fig. 11b); this yields $2^{j}$ different ways of decomposition.

In the case of wavelet packet transforms we have more freedom to choose the basic functions than in the case of wavelets. We shall choose a basis which in the best way matches the characteristics of the signal. In $\left[{ }^{29}\right]$ it is demonstrated that the best frequency resolution is achieved in the case of the Shannon entropy and for the local cosine transform (then the signal energy is concentrated just in a few waveforms).

The wavelet packet transform has been applied for processing signals in several papers $\left[{ }^{41-42}\right]$.

Usually the results obtained by the wavelet packet transform are interpreted in the time-frequency plane. A waveform is represented in this plane by a rectangle with its sides parallel to the time and frequency axes. According to the Heisenberg uncertainty inequality, the area of each rectangle must be smaller than $0.25 \pi\left[{ }^{41}\right]$. The amplitude of a waveform is encoded by darkening the rectangle in proportion to its waveform's energy. For example, the best basic time-frequency diagram of the Lorenz attractor (14) for $r=24.5$ is plotted in Fig. 12. For the decomposition level, $J=5$ has been taken. By increasing this number, the width of the frequency bands is decreased and more precise estimation of the actual motion is achieved. 


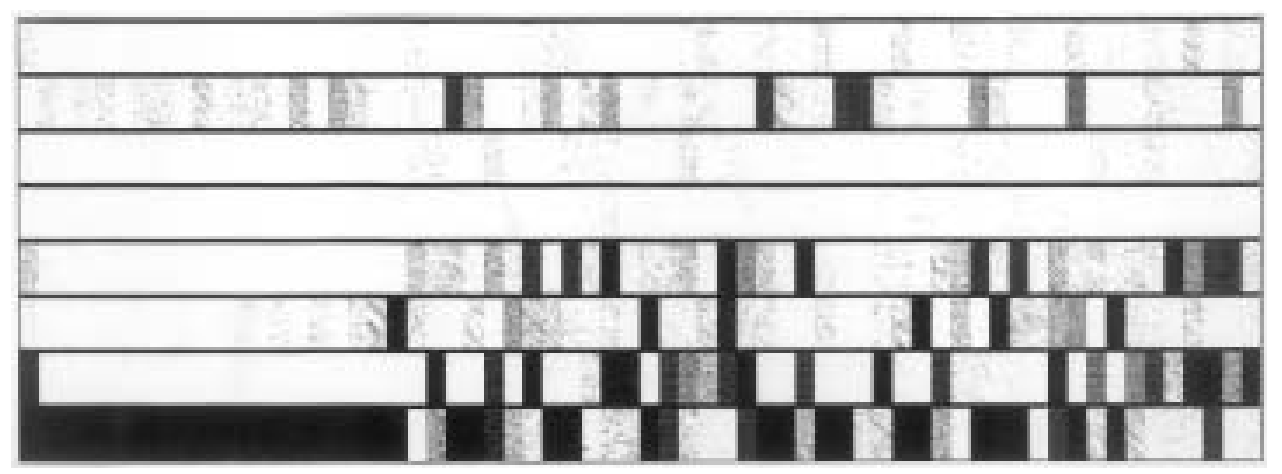

Fig. 12. Wavelet packet coefficients for the Lorenz equation (14), $r=24.5, \quad J=5$.

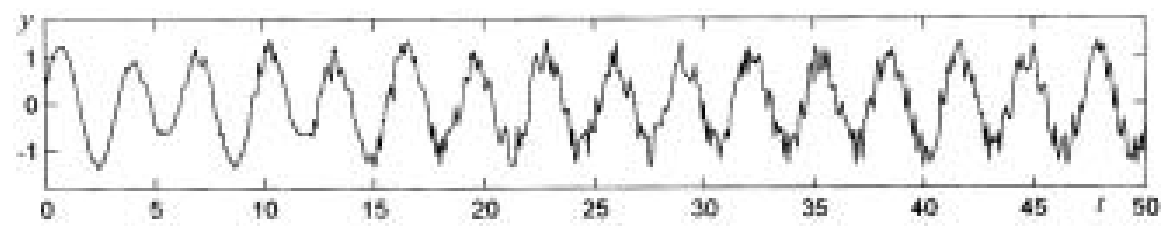

Fig. 13. Noisy vibrations described by the equation (26).

The wavelet packets are especially useful if we want to distinguish chaotic and random motion. For illustration we shall consider the motion

$$
y=\sin k t+\frac{f \cos \omega t}{k^{2}-\omega^{2}},
$$

which is a solution of the differential equation $y^{\prime \prime}+k^{2} y=f \cos \omega t$. Let us assume that $k=2, f=1$, and $\omega$ is a narrow-banded random variable $\omega=1+\alpha \xi(t)$, where $\xi(t)$ is a uniform zero-mean white noise (Fig. 13). The time-frequency diagram for $J=9$ in the case of the best basis is plotted in Fig. 14. It follows from this diagram that dark or grey rectangles can be found practically in all frequency bands (in the case of chaotic motions the wave energy is distributed between a finite number of decompositions).

\section{CONCLUSIONS}

There is much similarity in the classical Fourier method and the wavelet method $\left[{ }^{43}\right]$. The main difference between these two approaches is that Fourier technique analyses the signal over the whole domain and is unable to characterize completely its local behaviour. The wavelet method is indispensable for analysing signals presenting fast local variations such as transients or abrupt changes.

In this paper we tried to demonstrate that the wavelet technique is useful also for dedecting and exploring chaos in structural vibrations. 


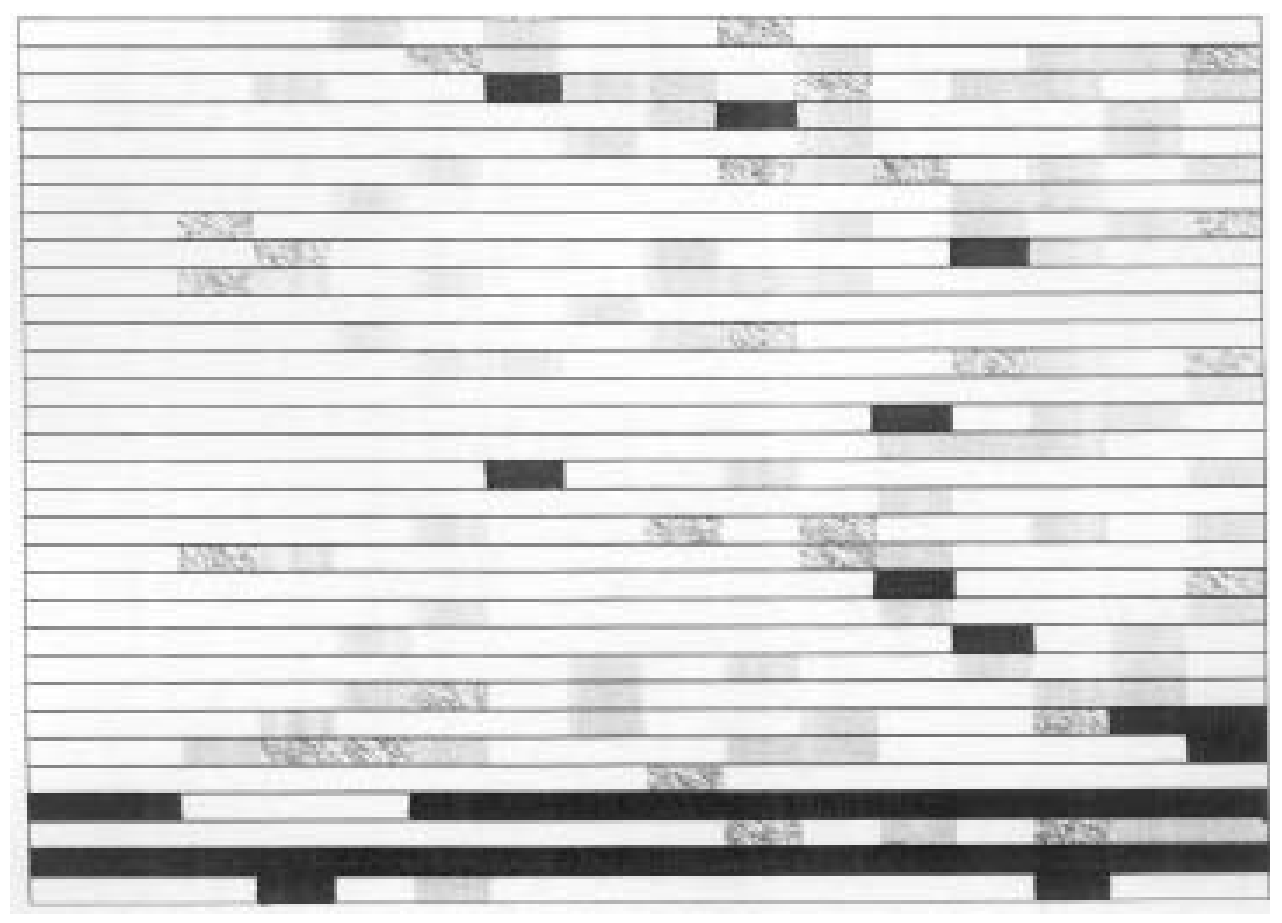

Fig. 14. Wavelet packet coefficients for the equation (26) when $J=9$.

\section{ACKNOWLEDGEMENT}

Financial support of the Estonian Science Foundation (grant No. 5240) is gratefully acknowledged.

\section{REFERENCES}

1. Newland, D. E. Introduction to Random Vibrations, Spectral and Wavelet Analysis. Longman, New York, 1993.

2. Chui, C. K. Wavelets: a Mathematical Tool for Signal Analysis. SIAM, Philadelphia, 1997.

3. Jaffard, S., Meyer, Y., and Ryan, R. D. Wavelets: Tools for Science and Technology. SIAM, Philadelphia, 2001.

4. Ghanem, R. and Romeo, F. A wavelet-based approach for model and parameter identification of non-linear systems. Int. J. Non-Linear Mech., 2001, 36, 835-859.

5. Jeong, H. Analysis of plane wave propagation in anisotropic laminates using a wavelet transform. NDT\&E Int., 2001, 34, 185-190.

6. Lepik, Ü. Application of wavelet transform techniques to vibration studies. Proc. Estonian Acad. Sci. Phys. Math., 2001, 50, 155-168.

7. Permann, D. and Hamilton, I. Wavelet analysis of time series for the Duffing oscillator: the detection of order within chaos. Phys. Rev. Lett., 1992, 69, 2607-2610.

8. Zheng Jibing, Gao Hangshan, and Guo Yinchao. Application of wavelet transform to bifurcation and chaos study. Appl. Math. Mech. (Shanghai, China), 1998, 19, 593-599. 
9. Wong, L. A. and Chen, J. C. Nonlinear and chaotic behavior of structural system investigated by wavelet transform techniques. Int. J. Non-Linear Mech., 2001, 36, 221-235.

10. Ioussoupov, V. I., Konkov, L. E., and Prants, S. V. Structural Hamilton chaos in coherent parametric atom-field interaction. Physica D, 2001, 155, 311-323.

11. Jubran, B. A., Hamdan, M. N., and Shabaneh, N. H. Wavelet and chaos analysis of flow induced vibration of a single cylinder in cross-flow. Int. J. Eng. Sci., 1998, 36, 843-864.

12. Huang, $\mathrm{X}$. and $\mathrm{Xu}, \mathrm{J}$. Unmasking chaotic mask by a wavelet multiscale decomposition algorithm. Int. J. Bifurcation Chaos, 2001, 11, 561-569.

13. Bukkapatnam, S. T. S., Kumara, S. R. T., Lakhtakia, A., and Srinivasan, P. The neighborhood method and its coupling with the wavelet method for signal separation of chaotic signals. Signal Process., 2002, 82, 1351-1374.

14. Ghanem, R. and Romeo, F. A wavelet-based approach for model and parameter identification of non-linear systems. Int. J. Non-Linear Mech., 2001, 36, 835-859.

15. Ribeiro, P. The second harmonic and the validity of Duffing equation for vibration of beams with large displacements. Comput. Struct., 2001, 79, 107-117.

16. Xiaoping Yuan. Lagrange stability for asymmetric Duffing equations. Nonlinear Analysis, 2001, 43, 137-151.

17. Moslehy, F. A. and Evan-Iwanowski, R. M. The effects of non-stationary processes on chaotic and regular responses of the Duffing oscillator. Int. J. Non-Linear Mech., 1991, 26, 61-71.

18. Konishi, K. Making chaotic behavior in a damped linear harmonic oscillator. Phys. Lett. A, 2001, 284, 85-90.

19. Bulsara, A. R., Jacobs, E. W., and Schieve, W. C. Noise effects in a nonlinear dynamic system: the superconducting quantum interference device. Phys. Rev. A, 1990, 42, 4614-4621.

20. Zhu, W. Q., Lu, M. Q., and Wu, Q. T. Stochastic jump and bifurcation of a Duffing oscillator under narrow-band excitation. J. Sound. Vibr., 1993, 165, 285-304.

21. Wei-Chan Xie. Effect of noise or chaotic motion of buckled column under periodic excitation. Nonlinear and Stochastic Dynamics, ASME Press, DE, 1994, 78, 215-225.

22. Lin, H. and Yim, S. C. S. Analysis of a non-linear system exhibiting chaotic, noisy chaotic and random behaviors. J. Appl. Mech., 1996, 63, 509-516.

23. Rong, H., Xu, W., and Fang, T. Principal response of Duffing oscillator to combined deterministic and narrow-band random parametric excitation. J. Sound. Vibr., 1998, 210, 483-515.

24. Haiwu, R., Wei, X., Guang, M., and Tong, F. Response of a Duffing oscillator to combined deterministic harmonic and random excitation. J. Sound. Vibr., 2001, 242, 362-368.

25. Liu, W. Y., Zhu, W. Q., and Huang, Z. L. Effect of bounded noise on chaotic motion of Duffing oscillator under parametric excitation. Chaos Solitons Fractals., 2001, 12, 527537.

26. Qiu, L. and Er, M. H. Wavelet spectrogram of noisy signals. Int. J. Electron., 1995, 79, 665677.

27. Szemplińska-Stupnicka, W. Nonstationary motions in stationary vibrating systems: a discussion. Dynamics and Vibration of Time-varying Systems and Structures. ASME Press, DE, 1993, 56, 421-425.

28. Misiti, M., Misiti, Y., Oppenheim, G., and Poggi, J.-M. Wavelet Toolbox for Use with Matlab. The Math Works. Inc., Natick, 1996.

29. Mathcad Extension Pack. MathSoft Internat., Surrey, 1999.

30. Rosso, O. A., Blanco, S., Yordanova, J., Kolev, V., Figliola, A., Schürmann, M., and Basar, E. Wavelet entropy: a new tool for analysis of short duration brain electrical signals. J. Neurosci. Methods, 2001, 105, 65-75.

31. Dai, Y. The time-frequency analysis approach of electric noise based on the wavelet transform. Solid-State Electron., 2000, 44, 2147-2153.

32. Scargle, J. D., Steiman-Cameron, T., Young, K., Donoho, D. L., Crutchfield, J. P., and Iamura, J. The quasi-periodic oscillations and very low frequency noise of Scorpius X-i as transient chaos: a dripping handrail? Astrophys. J., 1993, 411, L91-L94. 
33. Zu-Guo Yu and Vo Anh. Time series model based on global structure of complete genome. Chaos Solitons Fractals, 2001, 12, 1827-1834.

34. Percival, D. P. On estimation of the wavelet variance. Biometrica, 1995, 82, 619-631.

35. Ravier, P. and Amblard, P. O. Wavelet packets and de-noising based on higher-order-statistics for transient detection. Signal Process., 2001, 81, 1909-1926.

36. Manneville P. and Pomeau, Y. Intermittency and the Lorenz model. Phys. Lett., 1979, 75A, $1-2$.

37. Mittermayr, C. R., Lendl, B., Rosenberg, E., and Grasserbauer, M. The application of the wavelet power spectrum to detect and estimate $1 / \mathrm{f}$ noise in the presence of analytical signals. Analyt. Chim. Acta, 1999, 388, 303-313.

38. Wornell, G. W. Wavelet-based representations for the $1 / \mathrm{f}$ family of fractal processes. Proc. IEEE, 1993, 81, 1428-1450.

39. McWhirter, J. G. and Proudler, I. K., eds. Mathematics in Signal Processing V. Clarendon Press, Oxford, 2002.

40. Wickerhauser, M. V. Adapted Wavelet Analysis from Theory to Software. A. K. Peters, Wellesley, MA, 1994.

41. Cocchi, M., Seeber, R., and Ulrici, A. WPTER: wavelet packet transform for effecient pattern recognition of signals. Chemometr. Intell. Lab. Syst., 2001, 57, 97-119.

42. Ravier, P. and Amblard, P.-O. Wavelet packets and de-noising based on higher-order statistics for transient detection. Signal Process., 2001, 81, 1909-1926.

43. Perrier, V., Philipovitch, T., and Basdevant, C. Wavelet spectra compared to Fourier spectra. J. Math. Phys., 1995, 36, 1506-1519.

\section{Irregulaarsete võnkumiste ja kaose uurimine lainikute abil}

\section{Ülo Lepik}

Töö eesmärk on anda ülevaade lainikute rakendusvõimalustest irregulaarsete võnkumiste uurimisel. Selleks on kasutatud dekompositsiooni ning mitmeid numbrilisi karakteristikuid (energia jaotust erinevatel sagedustel, entroopiat, sarnasuse indeksit ja enesesarnasust). On analüüsitud müraga süsteemide käitumist ning käsitletud lainikute pakette, mille abil saab vähendada sagedusriba laiust ja eristada kaootilisi võnkumisi stohhastilistest. 